\title{
CASE STUDY: THERMOPLASTIC SCAPULOTHORACIC ORTHOSIS FOR TREATMENT OF WINGING SCAPULA
}

\author{
Steven Brown*, Gary Trexler \\ University of Oklahoma Health Science Center, Oklahoma City, Oklahoma, USA. \\ *E-mail: $\underline{\text { Steven-Brown@ouhsc.edu }}$
}

DOI: https://doi.org/10.33137/cpoj.v1i2.32043

\section{INTRODUCTION}

Winging scapula is a rare condition that can be painful and debilitating to the upper extremity involved 1 . This condition can affect the functional ability of the upper extremity, resulting in loss of range of motion, decreased power, and pain. ${ }^{1}$ The purpose of this study was to introduce and determine the clinical applicability of a custom thermoplastic scapulothoracic orthosis to aid in management of winging scapula. The thermoplastic scapulothoracic orthosis offers total contact and provides anterior-posterior compressive forces to stabilize the winging scapula. This design provides a semi-rigid structure that is lightweight and allows user adjustability. The study highlights the potential applicability of the custom thermoplastic scapulothoracic orthosis in the categories of pain, active range of motion at the shoulder, and overall self-reported activities of daily living.

\section{METHODS}

A 56-year-old male who experienced left winging scapula secondary to a traumatic work injury was evaluated and treated in the clinic. A custom scapulothoracic orthosis was designed, fabricated, and fit to the patient as part of his rehabilitation process. Prior to the fitting of the orthosis his pain levels were verbally discussed and the subject rated his pain on a scale of 0 to 10 , with $0=$ no pain, and $10=$ very much pain. Also, active range of motion was measured using a goniometer. After a six-week accommodation period these tests occurred again while wearing the orthosis and were compared. The subject stated that his pain levels had decreased his activities of daily living and caused him to live a more sedentary lifestyle.

\section{RESULTS}

The subject wore the scapulothoracic orthosis for approximately eight hours per day for six weeks. Selfreported pain was reduced in resting activity from $8 / 10$ to $3 / 10$. It was also reduced in glenohumeral flexion activities from 10/10 to 4/10. Active range of motion at the shoulder is listed in Table 1. Range of motion increased during shoulder flexion $106 \%$, abduction $63 \%$, internal rotation $55 \%$, and external rotation $61 \%$. The subject's winging scapula was visually reduced, but not completely eliminated. The subject verbally reported that the orthosis made activities such as cooking, typing on a computer, and brushing his hair/teeth more tolerable.

\begin{tabular}{|l|l|l|}
\hline Shoulder Motion & No Orthosis & Wearing Orthosis \\
\hline Flexion & 67 & 138 \\
\hline Abduction & 80 & 131 \\
\hline Internal Rotation & 42 & 65 \\
\hline External Rotation & 51 & 82 \\
\hline
\end{tabular}

Table 1. Active range of motion at the shoulder, with no orthosis and while wearing orthosis.

\section{CONCLUSION}

The use of a thermoplastic scapulothoracic orthosis in treatment of winging scapula secondary to a traumatic work injury had positive outcomes, including a reduction in pain levels, an increase in active range of motion and a reduction in visual scapular winging. The subject stated that with use of the orthosis, his activities of daily living increased. A limitation of the study includes the absence of an objective measurement technique used to measure scapular winging.

\section{SIGNIFICANCE}

The thermoplastic scapulothoracic orthosis is a viable option for treatment of winging scapula when surgery is not indicated or is not an option.

\section{REFERENCES}

1. Martin RM., Fish DE. Scapular winging: anatomical review, diagnosis, and treatments. Curr Rev Musculoskelet Med. 2008; 1(1), 1-11. doi: 10.1007/s12178-007-9000-5 